\title{
L'INFLUENCE DE L'ALIMENTATION DES VACHES LAITIERES SUR LA PRODUCTION DE LAIT DE BONNE QUALITÉ INDUSTRIELLE (1)
}

\author{
par \\ H. L. BÉRARD \\ B. A.-B. S. A. Ph. D. \\ J. M. ROSELL \\ M. D. \\ JULES TURGEON \\ B. S. A.
}

L'alimentation rationnelle des vaches laitières exerce une influence prépondérante tant sur la production quantitative que sur la production qualitative du lait destiné à des utilisations industrielles.

La valeur ou la qualité industrielle d'un lait peut s'exprimer par l'ensemble des propriétés et des caractéristiques physico-chimiques, biologiques et organoleptiques requises pour assurer la fabrication de produits laitiers de haute valeur commerciale. Pour donner une idée de la perfection industrielle pouvant être obtenue dans le domaine pratique, nous pouvons citer le beurre danois, les fromages suisses et français et autres produits laitiers de renommée mondiale. Nous pouvons avancer également que ces perfectionnements ont été rendus possibles par les progrès de la chimie et de la bactériologie du lait, par les soins apportés à la production et à la manipulation du lait, par l'amélioration des techniques de fabrication, par l'orientation rationnelle de l'alimentation des vaches laitières en vue de la production d'un lait de qualité supérieure et en dernier lieu, par l'application judicieuse de certaines méthodes de fertilisation et de conservation des récoltes.

Dans cet article, nous étudierons les questions relatives à l'amélioration de la qualité du lait et de ses produits dérivés par l'alimentation et par certaines méthodes de fertilisation et de conservation des récoltes, en mettant surtout en évidence les points suivants : l'influence des aliments sur la qualité du lait; les recherches faites et surtout les constatations pratiques établies après plus de cinquante années d'expériences dans les instituts d'expérimentation, dans les centres industriels laitiers et dans les associations coopératives laitières de Suisse, de France, d'Allemagne, du Danemark et des Etats-Unis; l'étude du règlement de production laitière paraissant le mieux connu et le plus solidement établi : le Règlement suisse, lequel a été utilisé dans bon nombre de pays après adaptation préalable à leurs conditions particulières.

Afin de faciliter notre exposé, nous avons subdivisé le sujet de la façon suivante :

(1) Travail reçu le 28 février 1936. 
A. Observation sur les influences des aliments sur la qualité du lait.

B. Observations sur les influences des aliments sur le beurre :

1. Sur la texture du beurre. 2 . Sur la qualité du beurre.

C. Observations sur les influences des aliments sur le fromage.

\section{A. OBSERVATIONS SUR LES INFLUENCES DES ALIMENTS SUR LA QUALITÉ DU LAIT}

L'alimentation constitue sans contredit une des plus importantes portes d'entrée, dans le lait, d'éléments qui ne devraient pas s'y rencontrer. En effet, depuis fort longtemps, on rattache à l'alimentation des vaches laitières un grand nombre d'anomalies dans le goût et l'odeur du lait. Il y a dans cette assertion une grande part de vérité. C'est ce que nous allons démontrer dans ce qui suit:

D'après des expériences connues de tous, nous savons que les plantes suivantes altèrent le goût et la saveur du lait : ail, ciboulette, poireau, armoise (saveur âcre et brûlante); raves (odeur sulfurée) ; euphorbe ou rhubarbe des pauvres (saveur et odeur désagréables et âcres); chicorée, paille moisie, navet, lupin (goût amer); trèfle et en général les légumineuses (goût et odeur de vaches ou de fourrages); collets de betterave (goût et odeur de pourri); pommes de terre germées (goût amer et désagréable); maïs (odeur et saveur fades); plantes bulbeuses à odeur forte (goût âcre et nauséabond) ; betterave et pulpes de betteraves fraîches (goût de poisson); pulpes de betteraves fermentées (goût et odeur d'acide butyrique, gon̂t toxique); les erucifères telles que chou, navet, etc., donnant le goût de la plante correspondante ou parfois le goût de moutarde. Selon les publications de Piex et Herschdoerfer (1) les goûts conférés au lait par les crucifères sont toxiques.

Ces mêmes auteurs (comme plusieurs autres d'ailleurs) font cependant quelques réserves au sujet des légumineuses dont les inconvénients sont assez faibles ; ils recommandent en plus, la constitution d'une ration à base de tourteaux (autres que ceux de crucifères), de grains concassés, de son, d'avoine, de fourrages divers, de carottes, de pois, de pommes de terre ainsi que l'usage de pulpes desséchées.

Dans presque tous les pays, il s'est fait un grand nombre de travaux élaborés sur ces questions et les conclusions obtenues se confirment l'une par l'autre. Il nous est impossible de citer toutes les expériences faites dans ce domaine, mais nous citerons quelques expériences relatées dans la littérature la plus accessible à notre continent.

HUNZIKer [2] rapporte que l'introduction dans la ration d'aliments mal conservés : foins, pailles, ensilage et grains moisis, de 
drèches de distillerie en fermentation ou mal conservées, etc., confère au lait et à ses dérivés des propriétés tout à fait indésirables et risque en plus d'altérer la santé de l'animal.

BABCock [3], dans ses expériences, arrive aux conclusions suivantes : le blé d'Inde vert, la plupart des concentrés vendus dans le commerce ainsi que quelques foins (du groupe des paturins du Canada, du Kentuckey et de Virginie) n'ont aucune mauvaise influence sur la saveur et l'arôme du lait. Par contre, il a énoncé les observations suivantes : a) l'ingestion de 30 livres de luzerne verte par vache, une heure avant la traite, produit dans le lait une saveur très prononcée. Cette constatation a été confirmée par LuOAS [4] et par Weaver, Kuhlman et Fouts $[5] ; b)$ le navet [6] donné à l'animal, une heure avant la traite, produit des odeurs indésirables dans le lait ; c) l'ingestion d'oignon et autres plantes bulbeuses analogues [7] se traduit, dès une minute après l'ingestion, par l'apparition du goût correspondant dans le lait; ce goût est encore apparent après 4 heures et il ne disparaît que si la traite est faite sept heures après l'ingestion ; d) un bidon de crème [8] possédant une saveur d'oignon assez prononcée peut communiquer cette saveur à un bassin entier de crème.

Dice [9], dans ses expériences sur le mélilot, a obtenu des résultats semblables à ceux de BABcock sur la luzerne.

Roadhouse et Konsther [10] ont démontré que le trèfle vert, ingéré moins de 5 heures avant la traite, provoque dans le lait une odeur anormale de vaches ou de fourrages.

ROSENGREN [11] a confirmé ces inconvénients du trèfle, en plus de les attribuer en général aux légumineuses. D'après d'autres expériences nous pouvons dire cependant que les inconvénients dus aux légumineuses disparaissent si la traite est faite assez longtemps après l'ingestion ou si le lait est pasteurisé et aéré.

Dorner [12] a démontré que les prairies artificielles à base de légumineuses communiquent au lait cette odeur particulière et qu'en revanche, il n'en est rien dans le cas de pâturage sur prairies naturelles à graminées dominantes.

Selon LERoy [13] les plantes contenant des produits ou principes toxiques - des alcaloïdes surtout - telles que les renoncules, les colchiques, la ciguë, les prêles (celles-ci sont fréquentes dans les prairies humides), etc., sont dangereuses pour la santé de l'animal et pour la qualité du lait. L'A. recommande alors de se méfier de la végétation poussant sur les levées de fossés et des prairies infestées de mauvaises herbes, et il préconise à cet effet, le drainage, les hersages ainsi que le ehaulage. Il recommande en plus d'éviter aux vaches l'ingestion d'aliments ayant subi la fermentation butyrique ou ne paraissant pas dans un bon état de conservation. 
Sur la betterave et ses produits dérivés, Post [14] a fait des travaux très intéressants et dans lesquels, il attribue les mauvais goûts produits par la betterave et ses produits, à la présence de bétaine ou triméthylglycocolle dans la betterave. Ce produit se transforme dans l'organisme même de l'animal (ou même avant si la betterave a subi une altération) en composés aminés tels que la triméthylamine, à odeur et saveur très désagréables. Il ne serait done pas nécessaire, d'après cet auteur, d'invoquer la fermentation éventuelle de la betterave pour expliquer les mauvais goûts qu'elle est régulièrement capable de communiquer au lait. Comme autre observation de cet auteur, les pulpes de betterave ensilées sans précautions spéciales sont une cause certaine de mauvais goûts, non seulement à cause de la triméthylamine, mais aussi à cause de l'acide butyrique si abondant dans les produits ensilés.

De nombreux médecins ont apporté la preuve irréfutable que les diarrhées infantiles d'automne et d'hiver [1] ont bien pour cause une mauvaise alimentation des vaches laitières (alimentation par betteraves, choux et certaines plantes citées plus haut). En particulier, PINARD [15] attribue principalement ces accidents à la betterave et aux pulpes de betterave fermentées. On ne dit pas cependant, si ces altérations peuvent persister après la pasteurisation du lait.

Comme considérations générales sur les crucifères, nous pouvons ajouter que ces plantes contiennent un glucoside susceptible de libérer, sous des influences diverses mais surtout bactériennes, du sulfocyanure ou thyocyanate d'allyle ou principe actif de l'essence de moutarde : facteur essentiel de certains mauvais goûts qui sont appelés goût de chou ou parfois goût de moutarde.

Orla-Jansen [16] a démontré en 1925, que ce glucoside ne donnait par lui-même aucun mauvais goût au lait. Avec les aliments frais, le mauvais goût n'apparaît que sous l'action de certaines bactéries provenant de l'eau, du fumier, etc.; elles agissent alors sur le glucoside éliminé dans le lait par l'animal.

Notons en passant que le sulfocyanure d'allyle n'est pas seulement la cause de mauvais goûts et de saveurs désagréables, mais il est également la cause d'accidents intestinaux graves ches les nourrissons [1] en raison de sa toxicocité. Pour des considérations citées plus haut, il serait peut-être bon de l'interdire complètement, en éliminant les quelques crucifères de la ration de l'animal.

Il est bon de rappeler ici que la plupart de ces odeurs et de ces saveurs se retrouveront dans la crème, le beurre et les fromages dans le cas d'utilisation industrielle de ce lait, surtout dans le cas de fabrication avec produits crus (non pasteurisés).

En plus des plantes utilisées régulièrement dans l'alimentation et susceptibles de conférer au lait des goûts et odeurs anormales, il 
faut compter aussi avec quelques mauvaises herbes et sur la contamination bactérienne de certains aliments.

Parmi les mauvaises herbes nuisibles dans ee domaine, nous pouvons inscrire sans hésitation les plantes suivantes: l'ail, l'oignon, le poireau, l'armoise, le tabouret des champs, l'herbe à poux, etc. Toutes ces mauvaises herbes, même lorsqu'ingérées en petites quantités (une demi-livre environ), communiqueront au lait et surtout à la crème des odeurs tout à fait répréhensibles et très difficiles à enlever. Généralement, dans les laiteries, on retourne à l'expéditeur tout produit laitier présentant ces saveurs. Advenant leur transformation en beurre, ce dernier obtiendra moins de $88 \%$ des points alloués et sera classé automatiquement comme un beurre de quatrième qualité. Pour enlever ces odeurs, il faut avoir recours à une aération et dégazéification intense de ces produits, laquelle est réalisée dans un bon nombre d'appareils commerciaux destinés à cette fin. Ces appareils, de même que Ia pasteurisation et l'aération ordinaire, enlèveront en même temps toutes les odeurs moins intenses (celles occasionnées par les légumineuses, etc.).

Comme mesure préventive, on recommande aux producteurs de lait de surveiller les heures d'alimentation et de traite. Par exemple, dans le cas de pacage des vaches, celles-ci seraient ramenées à l'étable le soir; de là, le lait de la traite du matin serait exempt des odeurs d'aliments. En plus, il faudrait ramener les vaches de trois à cinq heures avant la traite du soir. Cette mesure pouvant convenir assez bien aux producteurs importants, mais pour le fermier ordinaire elle n'est pas précisément économique. Le meilleur moyen de se débarrasser des odeurs dues aux mauvaises herbes, serait de détruire celles-ci par des pratiques culturales.

D'après les expériences précitées, il serait à recommander fortement, si on désire supprimer les odeurs anormales dans le lait, d'éviter l'introduction dans la ration d'aliments mal conservés (gelés, moisis, fermentés, etc.), ou d'aliments pouvant provoquer des troubles sérieux (quelques crucifères) et de prendre les mesures nécessaires pour détruire les plantes nuisibles (surtout les mauvaises herbes) et si possible, de surveiller les heures d'alimentation et de traite.

En plus des goûts anormaux produits par plusieurs aliments, nous pouvons en attribuer quelques-uns à des microorganismes qui, en raison de leur présence régulière sur ces aliments, ont acquis les propriétés plus ou moins phéno-typiques de produire dans le lait, le beurre et le fromage, des substances de métabolisme de goût et d'odeur semblables à ceux conférés par les plantes sur lesquels ils végètent: Les organismes producteurs de goûts variés tels que : goût de savon, goût de rance, goût amer, goût de poisson, goût de 
malt et même parfois des goûts aromatiques, sont surtout : Bact. saponatus, Bact. hervricola, Bact. tripholii, Bact. carotae, Bact. maltigenes, Torula amara, Bact. esterificans, Str. lactis maltigenes, Plectridium fotidum, etc. [17]. Dans le cas d'infection du lait par ces microorganismes, si la pasteurisation du lait est faite assez tôt, on ne notera aucune mauvaise répercussion sur le produit.

Les mesures précitées seraient suffisantes, en général, pour obtenir un lait sans saveur ou odeur anormale, mais nous devons ajouter aussi que l'exemption de saveur ou d'odeur particulière dans le lait, ne suffit pas toujours à la production de produits laitiers de haute qualité. En effet, quelques aliments, tout en altérant très peu les earactères organoleptiques du lait, contribueront cependant à en réduire les qualités beurrières et fromagères.

\section{B. OBSERVATIONS SUR LES INFLUENCES DES ALIMENTS SUR LE BEURRE}

1. Sur la texture du beurre. - Les principales recherches faites sur ce point, furent réalisées en Allemagne et dans les pays scandinaves. Nous pouvons citer en particulier, les travaux exécutés à l'Institut prussien de recherches laitières (Kiel, Allemagne) où l'on a publié des tableaux indiquant les variations de l'indice d'iode (mesure d'appréciation de la qualité et de la texture du beurre) après des essais très élaborés sur à peu près tous les types d'aliments et de rations utilisables.

Dans la suite, de nombreuses expériences ont été poursuivies dans à peu près tous les pays intéressés dans la production du beurre et les conclusions générales de ces travaux peuvent être résumées de la façon suivante :

L'alimentation s'est révélée comme un des facteurs les plus décisifs affectant la composition chimique, le point de fusion ou la texture de la matière grasse du lait et par là, le barattage de la crème (crème douce surtout) et finalement le malaxage du beurre. Nous pouvons dire que les aliments augmentant le pourcentage d'oléine dans la matière grasse du lait produisent un beurre mou; dans cette classe, nous pouvons inscrire les aliments suivants : tourteaux de lin, tourteaux de soja, paturins du Canada et du Kentuckey, gluten de maïs, avoine, son de blé, fourrages verts, etc. Par contre, les aliments diminuant le pourcentage d'oléine exerceraient une influence contraire : production de beurre trop ferme et trop dur. Dans cette dernière eatégorie, on peut inscrire en général les aliments riches en sucres, et en hydrates de carbone tels que : pommes de terre, blé d'Inde, ensilage de maïs, les betteraves, les tourteaux de coton, les foins, la luzerne, etc.

Leroy [13] classe les aliments au point de vue de leur influence 
sur la texture du beurre, selon la même base. Dans la première eatégorie, il inscrit les aliments donnant des beurres à point de fusion peu élevé et fournissant des produits de qualité en hiver et trop mous en été, comme exemple, les aliments augmentant le pourcentage d'oléine dans la matière grasse du lait. Dans l'autre, il fait rentrer les aliments donnant des beurres à point de fusion élevé, donnant des produits de bonne qualité en été, mais beaucoup trop durs en hiver et comme exemple, les aliments diminuant le pourcentage d'oléine. D'après le même auteur, les régimes alimentaires courants (à base de fourrages verts en été et d'ensilages et de pulpes de betterave en hiver) ont tendance à donner des beurres dont la qualité commerciale n'est pas en rapport avec la saison et il convient de modifier les propriétés spécifiques de ces régimes par l'addition de concentrés appropriés. Pour l'été, il serait préférable d'ajouter des tourteaux de coton à la ration et pour l'hiver, si on ne veut avoir de beurres trop durs, l'on se servira de tourteaux de lin et de son de blé.

Parmi les expériences faites sur le continent américain, nous pouvons citer celles d'Hunziker, Spitzer et Milus [18] lesquelles confirment les faits énoncés plus haut; celles de KeIth, KuHLMan WEAVER et GaLLuP [19] qui ont démontré que l'usage de tourteaux de coton (dans une proportion assez élevée dans la ration) augmentait le pourcentage de stéarine dans la matière grasse du lait; ce qui se traduisait par un barratage plus long de la crème et un malaxage du beurre plus difficile.

\section{Influences des aliments sur la qualité dú beurre.} Sur ce point, malgré le grand nombre d'observations pratiques, nous ne pouvons citer que quelques expériences relatées dans la littérature du continent américain. Ces expériences concernent plutôt les ensilages, les betteraves fourragères et demi-sucrières, les aliments mal conservés, etc., que les autres aliments utilisés régulièrement dans la ration.

D'après Eichstadt et Dibbern [20] certains aliments non susceptibles de provoquer par eux-mêmes l'apparition de mauvaises saveurs ou odeurs dans le lait, peuvent cependant exercer des actions défavorables sur le beurre.

PACr [21] démontre que les betteraves développent une action défavorable tant sur le rendement que sur les caractères organoleptiques du beurre, bien qu'il soit constaté que ce produit n'altère pas trop la saveur du lait lorsque donné à l'animal immédiatement après la traite. Selon le même auteur, les fourrages ensilés et, à un moindre degré, les betteraves peuvent altérer la prédisposition fer- 
mentative du lait jusqu'à le rendre inapte aux utilisations industrielles (tranformation en beurre ou en fromages).

D'après SAviNI [22] l'alimentation avec l'ensilage fait augmenter l'acidité du lait et entraîne en plus des variations dans sa valeur cryoscopique, laquelle est une des rares valeurs constantes de la composition du lait. Ceci démontrerait que l'équilibre physicochimique du lait subit des altérations et prouverait en même temps l'influence des aliments ci-haut nommés. Comme influences directes sur le beurre, SAvinI décrit des altérations légères des earactères organoleptiques, mais il prétend que ni l'ensilage ni les betteraves ne cunduisent à un produit très modifié au début; surtout si, dans la récolte du lait et dans la fabrication, on a suivi des méthodes rationnelles. Le beurre obtenu dans ees circonstances ne sera pas des plus parfumés, mais il n'aura pas non plus - dans les quelques jours suivant la fabrication - de saveurs répugnantes. Mais, selon l'A., l'influence de l'ensilage et de la betterave se ferait sentir surtout dans la conservation $d u$ beurre et à cela, s'ajouterait une altération plus importante : l'apparition de la saveur huileuse.

Les expériences de HESSELBERG [23] montrent également qu'une des causes du goût huileux dans le beurre serait à rechercher dans la betterave apportée dans l'alimentation des vaches laitières.

D'après les autres travaux publiés sur ces questions, nous pouvons dire que l'action des ensilages et des fourrages mal conservés, etc., se manifesterait plus ou moins longtemps après la fabrication, par la présence de goûts et d'odeurs variés et l'apparition de quelques défauts courants.

La meilleure preuve que nous puissions apporter à ces dires, c'est qu'en plusieurs pays, on permet l'usage de l'ensilage et des betteraves dans la ration lorsque le lait est destiné uniquement et sans interruption à la consommation directe, et que ces produits sont formellement interdits dans le cas de production du lait destiné à toute transformation industrielle.

\section{OBSERVATIONS SUR LES INFLUENCES DES ALIMENTS SUR LA QUALITÉ DU FROMAGE}

Il s'est fait sur cette question un grand nombre de publications et de travaux. La majeure partie des conclusions que l'on peut énoncer sur ce sujet serait le résultat de l'expérience pratique de plusieurs générations de fromagers de tous les pays d'Europe et des expériences systématiques faites surtout en Allemagne, en Suisse et en Italie. Nous relaterons tout d'abord quelques observations pratiques et finalement quelques expériences systématiques.

Il résulte [24] des recherches les plus récentes sur la composition chimique et sur les phénomènes biologiques du lait, que nous 
sommes encore bien loin de connaître tous les facteurs qui donnent au lait ses qualités fromagères. On peut cependant très bien concevoir que les modifications de tel ou tel fourrage, dues à une mauvaise conservation ou à une mauvaise préparation ou encore, à un changement de fumure (applications de purin sur les herbes à pâturages) puissent changer les propriétés chimiques et bactériologiques du lait au point d'affecter la composition et la fermentation du fromage.

Nous sommes un peu mieux renseignés sur l'influence de certains fourrages [24] sur les qualités fromagères du lait. Nous savons par exemple, que le fourrage ensilé ne se prête absolument pas à la production de lait de fromagerie parce qu'il l'infecte de bacilles butyriques. On sait également, que l'emploi intensif de tourteaux oléagineux cause une fermentation du lait qui provoque le gonflement sous presse et donne mauvais goût au fromage. En employant des fourrages inappropriés qui provoquent particulièrement la diarrhée chez les animaux, ou en fourrageant des farines, on infecte facilement le lait de bactéries du groupe $B$. Coli qui font gonfler le fromage et dont l'action peut encore être accrue par l'emploi de méthodes peu sanitaires dans la production et la fabrication. Il faut done choisir et employer les fourrages auxiliaires avec discernement, tant dans l'intérêt du producteur que du fromager.

Selon Peter [25] l'influence de l'affouragement sur la qualité du lait destiné à la fabrication de fromage est clairement établie dans quelques cas où le lait devient absolument impropre à toute fabrication de fromage. A titre d'exemple, il cite : l'affouragement d'herbe ensilée (gonflement butyrique) ou de fourrages qui provoquent une forte diarrhée chez l'animal (feuilles de betteraves, maïs vert, mélanges fourragers verts d'a.voine et de vesces (A. P. V.) ou encore de fourrages provoquant de fausses fermentations (fourrages fermentés ou décomposés).

Sur ce point, nous pouvons ajouter que la diarrhée chez la vache laitière est un des plus grands facteurs de contamination du lait et qu'elle est aussi en grande relation étiologique avec la mammite, pour laquelle elle paraît augmenter beaucoup la prédisposition.

Nous allons maintenant relater quelques expériences systématiques poursuivies sur cette question :

Dans ses expériences, Savini [22] démontre que l'influence et l'action des fourrages ensilés sur la fabrication de quelques types de fromages crus (erescenza et Bel Paese) d'après les expériences faites et les résultats obtenus, se résumeraient à ceci : $1^{\circ}$ la fabrication des fromages crus à maturation rapide est négativement influencée par une alimentation en fourrages ensilés ou en betteraves fourragères ; $2^{\circ}$ les fourrages ensilés endommagent davantage la fabrication fro magère que ne le font les betteraves fourragèrəz; $3^{3}$ ' 'in 
fluence, à conditions égales, est plus grande dans les fromages à longue maturation : ainsi les Bel-Paese (nécessitant plus de 40 jours de maturation) sont plus influencés que les types de fromage (crescenza) demandant 15 à 20 jours de maturation ; $4^{\circ}$ l'action se caractérise dans une première période par un retard dans l'apparition des caractères organoleptiques (odeur, saveur et arôme) et, dans une seconde période, par l'apparition dans les meules de véritables boursouflures, ce qui se traduit par de lourdes pertes pour les fabriques ; $5^{\circ}$ le manque de soins sanitaires et une mauvaise application des techniques de fabrication combinés à une alimentation défectueuse, produisent très facilement des produits défectueux. De tous ces exposés, SavinI conclut que dans la production d'un lait devant être transformé industriellement (en beurre ou en fromage) on ne saurait faire dominer dans la ration d'hiver des vaches laitières, les fourrages ensilés et les betteraves fourragères.

Steensberg, au Congrès international de Laiterie de Copenhague de 1931, a parfaitement bien démontré l'influence défavorable des déchets de betterave fermentés dans l'alimentation des vaches laitières.

KuRSthiner [26] dit que les bactéries propioniques et butyriques sont la cause de gonflement des fromages. Avec StaU B [27] il démontre que le lait provenant de vaches nourries avec des fourrages ensilés est impropre à la fabrication de l'Emmenthal de choix. Ils attribueraient ce fait à la présence des nombreuses bactéries butyriques contenues dans les fourrages ensilés.

BuRRI [28] énonce que la relation entre la fermentation butyrique des aliments et la fermentation butyrique du fromage ne fait pas de doute.

D'après les recherches de BuRRI et de KURSTEIner [29] le nombre de spores de bactéries butyriques (dues aux fourrages ensilés) est très considérable, variant entre 500 à 300.000 par gramme d'excrément et qu'un grand nombre de ces spores se retrouvent dans le lait. Ils concluent également, que les chances d'obtenir des fromages ne gonflant pas (l'Emmenthal surtout), à partir de lait provenant de vaches alimentées avee des fourrages ensilés, sont très minimes. Les mêmes auteurs prétendent que, même en récoltant le lait avec des soins que l'on ne peut prendre en pratique, le lait provenant de vaches nourries avec ces aliments est impropre à la fabrication de l'Emmenthal de qualité.

Selon Gorini [30] (voir aussi ses publications sur lait dysgénésique) l'ensilage de maïs préparé selon la méthode américaine garantit plus que tout autre une fermentation lactique prédominante, sans fermentation butyrique, et l'A. semble déduire qu'il n'y a aucune incompatibilité entre l'emploi de l'ensilage bien pré- 
paré et la fabrication du fromage Emmenthal. Néanmoins, il dit qu'il faut prendre en considération le fait que les ensilages ne sont pas toujours régulièrement parfaits et qu'ils favorisent l'infection nuisible du lait et l'apparition de fermentations gazeuses et de mauvaises saveurs. Sur ce point, nous pouvons ajouter qu'il serait très intéressant et utile de constater l'influence réelle de l'ensilage, tel qu'il est préparé dans la province de Québec, sur le fromage Cheddar et si possible, sur quelques autres types de fromages. Nous avons commencé des essais sur cette question afin de connaître l'influence de l'ensilage sur la fabrication et la conservation d'un type de fromage Bel Paese, qui sera fabriqué à Henryville par la Coopérative Fédérée de Québec.

Les analyses de lait que nous avons faites depuis quelques années en différentes régions de la province, nous ont donné, dans une proportion considérable, des résultats indiquant la plus ou moins grande valeur industrielle des laits examinés. Les cas de fermentations anormales (B. Coli, aerogenes), butyriques, caséolytiques étaient très nombreux.

Avec des laits d'une telle qualité, il serait presque impossible de vouloir réaliser la fabrication d'autres types de fromage que le Cheddar. Egalement, la fabrication de fromages affinés à pâte molle et semi-dure (Camembert, Brie, Bel Paese, Roquefort, Stilton) et de fromages frais à pâte molle et même cenx du type Gruyère, serait pratiquement impossible sans la pasteurisation du lait. Par cette dernière, on détruira la plupart des bactéries indésirables, mais il faudra toujours compter sur les fermentations butyriques. C'est pour eette raison que nous attirons l'attention des experts sur la nécessité de modifier quelques pratiques courantes d'alimentation.

Toutes les expériences précitées tendent surtout à démontrer l'importance des fermentations anormales des aliments sur la qualité des produits laitiers. Nous devons accorder une attention toute particulière à la fermentation butyrique, laquelle est presque toujours constatée dans les produits ensilés, ete.

A la base de la provocation des torts eonsidérables dans presque toutes les industries agricoles, les bactéries butyriques affectent tout particulièrement l'industrie du lait. L'action nuisible de ces bactéries est attribuable à l'odeur repoussante de l'acide butyrique produit et à la diffieulté de détruire ces bactéries introduites dans le lait. Toutes ces bactéries portent des spores et c'est à cela qu'elles doivent leur grande résistance à la chaleur : (elles résistent à la pasteurisation à $63^{\circ} \mathrm{C}$. pendant 30 minutes ; de plus, les spores résistent même à la stérilisation ordinaire). Ces propriétés thermo-résistantes nous font voir que la valeur de la pasteurisation est plutôt limitée lorsqu'il s'agit d'éviter leur aetion sur le beurre et les fromages. 
Si nous considérons l'apport considérable de ces bactéries de fermentations anormales dans le lait, il nous faut aussi considérer que les aliments mal conservés et surtout les ensilages sont les agents essentiels de cette introduction. En conséquence, il serait à recommander que les mesures préconisant le rejet de l'ensilage ou son a mélioration par différents procédés (le but de ces méthodes est de supprimer les fermentations indésirables) soient étudiées.

Cela ne veut pas dire que l'ensilage est un aliment destiné à disparaître de l'alimentation des vaches; depuis une quinzaine d'années, il s'est fait beaucoup de travail pour arriver à produire un ensilage hygiénique et pouvant convenir sans danger aux utilisations industrielles du lait.

Le principe de quelques méthodes d'amélioration des ensilages est le suivant : par des solutions acides (acides sulfurique, chlorhydrique dilués, lait écrémé ou petit-lait acidifié par des ferments lactiques) on abaisse le $p \mathrm{H}$ de l'ensilage à un point tel que les microorganismes indésirables ne se développent plus; cet abaissement du $p \mathrm{H}$ ayant pour but la production d'une fermentation lactique prédominante dans le produit. Les principales méthodes répandues en Europe sont les suivantes : $a$ ) la méthode d'ensilage lactique, préconisée en Allemagne et en Italie et adaptée en France, consistant en l'arrosage des couches d'ensilage, lors du remplissage, avec une quantité déterminée de lait écrémé acidifié par des ferments lactiques. Plusieurs instituts d'Europe s'occupent de la distribution de ces cultures et il semble qu'avant longtemps cette mesure sera rendue obligatoire en France, si on en juge par les vœux émis par le Conseil supérieur d'Hygiène de France; b) par contre, dans, la méthode Virtanem (ensilage A. I. V.) décrite dans la Revue "Le Lait" 13, 1933, p. 315-339, et dans le "Journal of Dairy Science ", janvier 1935, p. 63, l'abaissement du $p \mathrm{H}$ est tel qu'il empêche toute action enzymatique ou bactérienne dans le produit.

D'après les expériences faites, ces ensilages n'auraient pas de mauvaises influences sur la qualité des produits laitiers et, de plus, les principes nutritifs de ces produits seraient conservés pratiquement intacts. Ceci serait déjà un avantage appréciable, car tout le monde connaît les pertes importantes d'éléments nutritifs subies au cours de la préparation ordinaire de l'ensilage.

Toutes ces considérations militent en faveur d'expériences étendues sur ces méthodes d'amélioration de la qualité des ensilages et permettraient en même temps la suppression d'une des causes les plus sérieuses d'altérations du beurre et des fromages.

Voici maintenant comment ont été appliquées dans la pratique les conclusions de tous ces travaux de recherches et d'expérimen- 
tation. A cet effet, nous allons reproduire textuellement quelques articles $d u$ règlement suisse de production laitière.

Tous les articles qui vont suivre [31] sont obligatoires pour tous les producteurs et les entreprises qui livrent du lait et des produits laitiers au commerce en Suisse.

\section{RÈGLEMENT SUISSE DE PRODUCTION LAITIÈRE (1 ${ }^{\text {cr }}$ juin 1934).}

1. Traitement des fourrages et affouragement.

\section{A. Ce régime vert.}

Article 7. - Le régime vert sera appliqué de façon à éviter les troubles digestifs qui, accompagnés d'une plus ou moins forte diarrhée, compromettent non seulement la qualité du lait, mais aussi dans une forte mesure le rendement économique et les fonctions de reproduction des vaches laitiè res.

Article 9. - Le meilleur fourrage vert pendant la stabulation est une bonne herbe (ni trop jeune ni trop vieille) provenant de bonnes prairies naturelles, de champs de trèfle ou d'autres prairies artificielles bien cultivées et bien fumées. Eviter tout régime unilatéral, spécialement l'emploi exclusif de jeune trèfle, d'herbe de vergers ou de prairies trop intensivement arrosées au purin. On préviendra les dangereuses perturbations de fabrication en donnant aux vaches laitières des mélanges appropriés de différents fourrages verts.

Le seigle, le maïs et l'avoine verts, les vesces, le colza, la spergule, les feuilles de betteraves (fourragères et sucrières) et de choux-raves (navets) ne doivent être donnés qu'à l'état absolument frais, en quantités modérées et seulement on mélange avec de l'herbe fraîchement fauchée ou du fourrage sec.

Article 10. - Sont autorisés comme compléments du fourrage vert :

a) Du fourrage sec. Il est toujours recommandable de donner du foin avec l'herbe très mouillée ou qui peut provoquer la diarrhée.

b) Quand le temps est froid et pluvieux : un peu de bon gros son de blé (éventuellement avec le sel nécessaire) donné sec, dans la crèche ou à la main.

Article 11. - Il est interdit de donner pendant le régime vert, tout fourrage qui n'est pas mentionné aux articles 9 et 10 .

En temps de pénurie de fourrages verts appropriés, l'acheteur de lait et le producteur s'entendront pour compléter éventuellement la ration avec des céréales concassées, du son, etc.

Article 12. - Sont spécialement exclus de l'affouragement des vaches laitières :

a) L'herbe arrosée au purin, dont la eroissance est trop avancée, ou sur laquelle, on a épandu des engrais chimiques après le départ de la végétation.

b) L'herbe qui fermente (échauffée).

c) L'herbe en voie de décomposition à la suite de pluies persistantes.

d) Les déchets de jardinage de toute sorte, les pommes de terre et leurs tiges, les fruits et leurs résidus.

e) Le foin ou le regain en fermentation.

B, Le régime sec.

Article 14. - En choisissant les fourrages, on tiendra compte tout d'abord 
de leur composition. Ils doivent être sains, exempts de substances toxiques ou nuisibles à la santé. Sont particulièrement dangereux les fourrages rancis, moisis, acides, attaqués par des parasites animaux ou végétaux ou détériorés par la fermentation.

Article 15. - La base de la ration sera composée de foin et de regain sain ayant normalement fermenté, exempts de moisissures ; exceptionnellement de bonne paille.

On ne donnera pas de foin ou de regain bruni ou moisi aux vaches laitières. Entrent en ligne de compte comme fourrages complémentaires :

a) La balle de céréales.

b) Les betteraves fourragères et demi-sucrières, les carottes et les chouxraves en quantités modérées.

c) Les pommes de terres saines, fraîchement cuites à la vapeur, puis refroidies et données entières.

Article 16. - On ne pourra compléter la ration de foin, de regain ou de paille qu'avec des fourrages concentrés sains, digestibles, bien préparés et en mélange judicieux. Sont admis à ce titre :

a) Les grains de céréales sains, fraîchement concassés, comme l'orge, l'épeautre, l'avoine, le blé et le son de blé.

Le seigle et la farine fourragère fraîche ne peuvent être employés qu'en petites quantités et seulement d'entente avec l'acheteur de lait, lorsqu'il s'agit de lait de fromagerie.

b) Les tourteaux oléagineux frais (lin, arachide) en quantité ne dépassant pas un demi kilogramme par tête et par jour pour la moyenne du troupeau. En aucun cas, la ration de tourteaux d'une seule vache ne doit dépasser 1 kilogramme.

c) Les germes d'orge, le malt desséché, les cossettes de betteraves desséchées, mélangées avec d'autres concentrés. La ration de chacun de ces fourrages ne dépassera pas un demi kilogramme par vache et par jour.

La ration totale de tous les fourrages concentrés ne doit pas dépasser 1 kilogramme et demi par vache et par jour.

Les tourteaux ne doivent jamais être donnés seuls, mais en mélange avec du grain concassé ou du son de blé.

Normalement, on ne donnera pas plus de 30 grammes de sel ( 1 once environ) par vache et par jour.

Article 17. - Il ne faut en aucun cas mouiller les fourrages concentrés ou les donner sous forme de breuvage (barbotages). On les conservera dans des locaux frais, secs et aérés. Les récipients servant à l'affouragement des concentrés ne doivent pas être laissés à l'étable.

\section{Dispositions communes aux deux régimes (vert et sec).}

Article 18. - Les fourrages suivants sont interdits :

a) Les fourrages ensilés de toute sorte (ensilage doux, de maïs, de feuilles de betteraves, ete.

c) Les pommes de terre crues et les racines de toute sorte en voie de décomposition.

c) Les cossettes de betteraves frâ̂ches ou desséchées et longtemps trempées, les 
fourrages mélassés de toute sorte, les germes d'orge longtemps trempés, les tourteaux trempés, le malt frais, la levure de bière, les résidus de la distillation des pommes de terre (vinasses) et de la fabrication des levures.

d) Las fruits, les mares et les déchets de fruits de toute sorte.

e) Les fourrages múlangés du commerce de composition inconnue.

f) Tous les autres fourrages qui ne sont pas autorisés d'après les articles précédents.

Article 19. - La division de l'agriculture du Département fédéral de l'économie publique peut autoriser des exceptions temporaires aux dispositions de l'article 18, dans le cas de pénurie de certains aliments et sur l'avis des autorités compétentes.

2. FuMURe des terRes destinées A LA CULTURE FoURRAGìre.

Article 2. - On appliquera le fumier et les engrais chimiques nécessaires à la culture des prairies arțificielles (plantes annuelles, trèfles, mélanges, etc.) autant que possible à la récolte précédente, c'est-à-dire qu'on les enfouira en ensem эnçant.

Article 3. - On épandra les engrais chimiques sous quelque forme que ce soit exclusivem nt pendant le repos de la végétation, c'est-à-dire depuis l'arrière-automne jusqu'au commencement du printemps.

Article 4. - On restreindra autant que possible l'emploi du purin que l'on répartira sur toutes les prairies, afin d'éviter la croissance de fourrages médiocres (herbe forcée ou envahie de mauvaises herbes).

Il est interdit d'épandre le purin sur de l'herbe dont la végétation est trop avancée. Le purin doit être fortement dilué avec de l'eau. Il est interdit d'ajouter quoi que ce soit au purin pour le conserver, sauf de l'eau.

\section{Autres dispositions du règlemsnt suisse.}

Dans l'article 20, on dit : Il est interdit de loger des pores et des poules dans les étables de vaches laitières, à moins de les séparer suffisamment. Dans l'article 21, on dit : Eviter la litière verte de toute sorte, telles que l'herbe, les tiges de pommes de terre, etc. La litière de tourbe n'est permise que comme absorbant dur purin. Les matériaux à faible pouvoir absorbant seront mólangés à la litière.

Pendant la traite, il est interdit de sortir le fumier, de panser les animaux ou d'exécuter tout travail qui soulève de la poussière.

Dans l'article 28, on dit : Sont particulièrement exclus de la livraison et de l'alim эntation :

a) Le lait qui forme des grum эaux à la cuisson ou au repos, révélant généralem эnt une maladie du pis.

d) Le lait de vaches atteintes de maladies graves ou auxquelles on administre des médicaments et ce, pendant les 4 jours qui suivent leur dernier emploi.

e) Le lait de vaches partiellement taries que l'on ne trait pas plus d'une fois par jour.

h) Le lait qui présente des défauts graves de coagulation à l'essai de présure. 


\title{
BIBLIOGRAPHIE
}

[1] Pien et Herschdoerfer. Le Lait, 1935, p. 1 et p. 257.

[2] HUNZIKER. Butter Industry, $2^{\mathrm{e}}$ édition.

[3] Bавсоск. U. S. Dep. Agric., Bull 1190, 1932.

[4] LuCAs. Michigan Exp. Stat. Quart., Bull. XII, 1, 1929.

[5] Weaver, Kuhlman et Fouts. J. Dairy Science, janvier 1935, p. 55.

[6] Baвсоск. U. S. Dep.. Agric., Bull. 1208, 1923.

[7] Вавсоск. U. S. Dep. Agric., Bull., 1326, 1925.

[8] Вавсоск. U. S. Dep. Agric. Tech., Bull., no 9, 1927, p. 6.

[9] Dice. North Dakota. Biennium Rept., Bull. 1934, 1926.

[10] Roadhouse et Koestler (cité par Dornic). Le Lait, 1930, p. 100.

[11] Rosengrein. Svenska Mejeritidingen, $\mathrm{n}^{\circ} 26,1927$.

[12] Dorner. Le Lait, janvier 1930, p. 100.

[13] Leroy, Le Lait, 1925, p. 286.

[14] Post. Pharm. Weekblad., t. LXVII, 1930, p. 1309.

[15] Renault. Conseil Sup. d'Hygiène de France.

[16] OrLA-Jensen. Le Lait, janvier 1925, p. 30.

[17] Rosell. Canadian Dairy and Ice Cream Journal, soût 1933.

[18] Hunziker, Spitzer et Mills. Purdue Bull., 159, 1912.

[19] Keith-Kuhlman-Weaver et Gallup. Journal Dairy Sc., 1935, p. 469.

[20] Eichstadt et Dibbern. Milchwirsch. Forsch., t. VI, 1928, p. 564.

[21] PaCr. Le Lait, juillet-août 1934, p. 742.

[22] Savini. Annali dell' Istituto Sperimentale di Caseificio di Lodi, Vol. VI, no $7-8$, septembre 1933 , p. 273.

[23] Hesselberg. Le Lait, 1925, p. 913.

[24] Règlement de livraison du lait de la Suisse romande.

[25] Peter. Conférence nationale de l'Industrie laitière, Berne, 1935, p. 21.

[26] Kursteiner. Conférence nationale de l'Industrie laitière, Berne (Suisse), p. 39 .

[27] Kursteiner et Staub. Schweizerische Milchzeitung, no 95-97, 1921.

[28] BURri. Congrès international de Laiterie, octobre 1923.

[29] Burri et Kursteiner. Le Lait, 1922, p. 748.

[30] GoRINI. Congrès international de Laiterie, octobre 1923.

[31] Règlement suisse de production du lait, Imprim orie Fédérale, Berne (Suisse).

\section{REVUE}

\section{LA CASÉINE COMME MATIÈRE PREMIÈRE POUR L'INDUSTRIE CHIMIQUE}

\author{
par \\ G. GÉNIN
}

Il y a quelques semaines, M. Rowe a fait devant l" "Oil and Colour Chemists' Association" de Londres, une communication 